\title{
ON THE STRUCTURE AND INVARIANCE OF THE BARNETT-LOTHE TENSORS*
}

\author{
BY \\ P. CHADWICK (University of East Anglia, Norwich, England) \\ AND
}

T. C. T. TING (University of Illinois at Chicago)

\begin{abstract}
The three real tensors introduced by Barnett and Lothe into the theory of steady plane motions of an anisotropic elastic body are shown to have algebraic representations the structure of which is largely independent of material symmetry. The allied form of the complex impedance tensor central to the analyses of surface and interfacial waves in anisotropic elastodynamics is also obtained. A detailed study of the representations yields alternative routes to known results and a variety of new relations. The paper concludes with a discussion of the invariance properties of quantities appearing in the representations under rotations of the reference frame about the direction in which the deformation is uniform.
\end{abstract}

1. Preliminaries. We are concerned in this paper with an elastic body $\mathscr{B}$ which is homogeneous and of general anisotropy with respect to a natural reference configuration $\mathscr{R}$. The discussion relates to small-amplitude motions of $\mathscr{B}$ which are plane and steady in the sense that the displacement from $\mathscr{R}$ is a function of $x_{1}-v t$ and $x_{2}$ only, $x_{1}$ and $x_{2}$ belonging to a fixed rectangular Cartesian coordinate system $x_{i}, i=1,2,3$, and $t$ being the time. The speed $v$ is regarded as a real parameter.

In the theory of steady plane elastodynamics a basic role is played by three real tensors, first brought into a unified formalism by Barnett and Lothe [1], and a complex impedance tensor introduced by Ingebrigtsen and Tonning [2]. For convenience we refer throughout to a review article by Chadwick and Smith [3] for background information and references to the original papers. The notations $\mathbf{S}_{1}, \mathbf{S}_{2}, \mathbf{S}_{3}$, and $\mathbf{Z}$ for the Barnett-Lothe and impedance tensors adopted in [3] are followed here, but we take note of the alternatives $\mathbf{S}_{1}=\mathbf{S}, \mathbf{S}_{2}=-\mathbf{Q}, \mathbf{S}_{3}=-\mathbf{B}$ and $\mathbf{S}_{1}=\mathbf{S}, \mathbf{S}_{2}=\mathbf{H}, \mathbf{S}_{3}=-\mathbf{L}$ preferred by Barnett and Lothe $[1,4]$ and Ting [5], respectively. The following facts are sufficient for our immediate purposes. First, $\mathbf{S}_{1}, \mathbf{S}_{2}, \mathbf{S}_{3}$, and $\mathbf{Z}$ are tensor-valued functions of the speed $v$ defined on

\footnotetext{
* Received February 10, 1986.
} 
the half-open interval $I=\{v: 0 \leqslant v<\hat{v}\}, \hat{v}$ being the limiting speed [3, Sec. VI, B]. Second, $\mathbf{S}_{2}$ is symmetric and, subject to the linear elasticity tensor in $\mathscr{R}$ of the material composing $\mathscr{B}$ being strongly elliptic, positive definite $[3$, Sec. IV, D]. Third, the relations

$$
\begin{aligned}
\mathbf{S}_{1} & =-\mathbf{S}_{2} \mathbf{S}_{1}^{\mathrm{T}} \mathbf{S}_{2}^{-1}, \\
\mathbf{S}_{3} & =-\mathbf{S}_{2}^{-1}\left(\mathbf{I}+\mathbf{S}_{1}^{2}\right), \\
\mathbf{Z} & =\mathbf{S}_{2}^{-1}\left(\mathbf{I}+i \mathbf{S}_{1}\right)
\end{aligned}
$$

connect the four tensors, I being the identity tensor and $T$ indicating transposition. It follows from (1) that $\mathbf{S}_{1} \mathbf{S}_{2}$ and $\mathbf{S}_{2}^{-1} \mathbf{S}_{1}$ are skew-symmetric and then from (2) and (3) that $\mathbf{S}_{3}$ is symmetric and $\mathbf{Z}$ Hermitian [3, Secs. IV, D, VII, D].

As in the preceding paragraph, dependence on $v$ will be left implicit and, unless qualified to the contrary, results are understood to hold in the subsonic interval $I$. The symbol $\otimes$ denotes the tensor product of two vectors; the trace and determinant of a tensor are abbreviated to $\operatorname{tr}$ and det; and the principal invariants $I_{\mathbf{A}}, I I_{\mathbf{A}}, I I I_{\mathbf{A}}$ of an arbitrary tensor $\mathbf{A}$ are defined by

$$
I_{\mathbf{A}}=\operatorname{tr} \mathbf{A}, \quad I I_{\mathbf{A}}=\frac{1}{2}\left\{(\operatorname{tr} \mathbf{A})^{2}-\operatorname{tr} \mathbf{A}^{2}\right\}, \quad I I I_{\mathbf{A}}=\operatorname{det} \mathbf{A} .
$$

2. Representation of $\mathbf{S}_{\mathbf{1}}$. In view of the positive definiteness of $\mathbf{S}_{2}$ it follows from (1), on taking the trace and determinant of each side, that

$$
I_{\mathbf{S}_{1}}=\operatorname{tr} \mathbf{S}_{1}=0, \quad I I I_{\mathbf{S}_{1}}=\operatorname{det} \mathbf{S}_{1}=0 .
$$

The characteristic equation of $\mathbf{S}_{1}$ is thus

$$
\operatorname{det}\left(\nu \mathbf{I}-\mathbf{S}_{1}\right) \equiv \nu^{3}+I I_{\mathbf{S}_{1}} \nu=0,
$$

with

$$
I I_{\mathrm{S}_{1}}=-\frac{1}{2} \operatorname{tr} \mathbf{S}_{1}^{2}
$$

Put $\mathbf{W}=\mathbf{S}_{1} \mathbf{S}_{2}, \mathbf{S}=\mathbf{S}_{2}^{-1}$. Then, as mentioned above, $\mathbf{W}$ is skew-symmetric and can be expressed as

$$
\mathbf{W}=\omega(\mathbf{r} \otimes \mathbf{q}-\mathbf{q} \otimes \mathbf{r}),
$$

where $\mathbf{q}$ and $\mathbf{r}$ are members of an orthonormal basis $\{\mathbf{p}, \mathbf{q}, \mathbf{r}\}$ [6, p. 29]. Hence

$$
\mathbf{S}_{1}=\mathbf{W S}=\omega\{\mathbf{r} \otimes(\mathbf{S q})-\mathbf{q} \otimes(\mathbf{S r})\}
$$

and, from (6),

$$
I I_{\mathbf{S}_{1}}=\omega^{2}\left[\{\mathbf{q} \cdot(\mathbf{S q})\}\{\mathbf{r} \cdot(\mathbf{S r})\}-\{\mathbf{q} \cdot(\mathbf{S r})\}^{2}\right] .
$$

The terms in square brackets in (7) form a $2 \times 2$ principal minor of the matrix representation of $\mathbf{S}$ relative to $\{\mathbf{p}, \mathbf{q}, \mathbf{r}\}$ and are accordingly positive, as is $I I_{\mathbf{S}_{1}}$. We infer from $(5)_{2}$ that the eigenvalues of $\mathbf{S}_{1}$ are $\pm i s, 0$ where $s$ is real and, without loss of generality, nonnegative. We suppose henceforth that $s>0$. An alternative proof that the eigenvalues of $\mathbf{S}_{1}$ are $\pm i$ s, 0 can be obtained by setting $\mathbf{W}=\mathbf{S}_{1} \mathbf{S}_{2}, \mathbf{D}=\mathbf{S}_{2}$ in the Appendix of [5]. 
Since $\mathbf{S}_{1}$ is a real tensor, eigenvectors associated with the eigenvalues $\pm i s, 0$ are of the form $\mathbf{a} \pm i \mathbf{b}, \mathbf{c}$ where $\mathbf{a}, \mathbf{b}, \mathbf{c}$ are real vectors. We deduce from the eigenrelations

$$
\mathbf{S}_{1}(\mathbf{a} \pm i \mathbf{b})= \pm i s(\mathbf{a} \pm i \mathbf{b}), \quad \mathbf{S}_{1} \mathbf{c}=\mathbf{0}
$$

that

$$
\mathbf{S}_{1} \mathbf{a}=-s \mathbf{b}, \quad \mathbf{S}_{1} \mathbf{b}=s \mathbf{a}, \quad \mathbf{S}_{1} \mathbf{c}=\mathbf{0} .
$$

If the vectors $\mathbf{a}_{0} \pm i \mathbf{b}_{0}, \mathbf{c}_{0}$ satisfy (8), so do $(\alpha \pm i \beta)\left(\mathbf{a}_{0} \pm i \mathbf{b}_{0}\right), \gamma \mathbf{c}_{0}$ where $\alpha, \beta, \gamma$ are arbitrary real numbers such that $\alpha^{2}+\beta^{2} \neq 0, \gamma \neq 0$. If $\mathbf{a}_{0}, \mathbf{b}_{0}, \mathbf{c}_{0}$ is a particular solution of (9), the general solution is therefore

$$
\mathbf{a}=\alpha \mathbf{a}_{0}-\beta \mathbf{b}_{0}, \quad \mathbf{b}=\beta \mathbf{a}_{0}+\alpha \mathbf{b}_{0}, \quad \mathbf{c}=\gamma \mathbf{c}_{0} .
$$

On account of the distinctness of the eigenvalues, $\mathbf{a}_{0}, \mathbf{b}_{0}, \mathbf{c}_{0}$, and hence $\mathbf{a}, \mathbf{b}, \mathbf{c}$, are linearly independent.

The vectors $\mathbf{a}^{\prime}, \mathbf{b}^{\prime}, \mathbf{c}^{\prime}$ reciprocal to $\mathbf{a}, \mathbf{b}, \mathbf{c}$ are defined by

$$
\mathbf{a}^{\prime}=g^{-1} \mathbf{b} \wedge \mathbf{c}, \quad \mathbf{b}^{\prime}=g^{-1} \mathbf{c} \wedge \mathbf{a}, \quad \mathbf{c}^{\prime}=g^{-1} \mathbf{a} \wedge \mathbf{b},
$$

with

$$
g=[\mathbf{a}, \mathbf{b}, \mathbf{c}] .
$$

The definitions (11) and the associated identity

$$
\mathbf{a} \otimes \mathbf{a}^{\prime}+\mathbf{b} \otimes \mathbf{b}^{\prime}+\mathbf{c} \otimes \mathbf{c}^{\prime}=\mathbf{I},
$$

are used frequently, and without explicit mention, in the sequel.

Proceeding from Eqs. (9) we now obtain the representation

$$
\begin{aligned}
\mathbf{S}_{1} & =\left(\mathbf{S}_{1} \mathbf{a}\right) \otimes \mathbf{a}^{\prime}+\left(\mathbf{S}_{1} \mathbf{b}\right) \otimes \mathbf{b}^{\prime}+\left(\mathbf{S}_{1} \mathbf{c}\right) \otimes \mathbf{c}^{\prime} \\
& =s\left(\mathbf{a} \otimes \mathbf{b}^{\prime}-\mathbf{b} \otimes \mathbf{a}^{\prime}\right)
\end{aligned}
$$

of $\mathbf{S}_{1}$. It follows from Eqs. (10) and their reciprocals,

$$
\begin{aligned}
& \mathbf{a}^{\prime}=\left(\alpha^{2}+\beta^{2}\right)^{-1}\left(\alpha \mathbf{a}_{0}^{\prime}-\beta \mathbf{b}_{0}^{\prime}\right), \\
& \mathbf{b}^{\prime}=\left(\alpha^{2}+\beta^{2}\right)^{-1}\left(\beta \mathbf{a}_{0}^{\prime}+\alpha \mathbf{b}_{0}^{\prime}\right), \\
& \mathbf{c}^{\prime}=\gamma^{-1} \mathbf{c}_{0}^{\prime},
\end{aligned}
$$

that, for all allowable values of $\alpha, \beta, \gamma$,

$$
\mathbf{a} \otimes \mathbf{b}^{\prime}-\mathbf{b} \otimes \mathbf{a}^{\prime}=\mathbf{a}_{0} \otimes \mathbf{b}_{0}^{\prime}-\mathbf{b}_{0} \otimes \mathbf{a}_{0}^{\prime}
$$

This confirms that, in (12), $\mathbf{a} \otimes \mathbf{b}^{\prime}-\mathbf{b} \otimes \mathbf{a}^{\prime}$, as well as $s$, is uniquely determined by $\mathbf{S}_{1}$.

The result of squaring (12) is

$$
\mathbf{S}_{1}^{2}=-(1-F)\left(\mathbf{a} \otimes \mathbf{a}^{\prime}+\mathbf{b} \otimes \mathbf{b}^{\prime}\right),
$$

where

$$
F=1-s^{2}=1-I I_{\mathbf{S}_{1}}=1+\frac{1}{2} \operatorname{tr} \mathbf{S}_{1}^{2} .
$$

The fact that the right-hand side of (14) is in spectral form demonstrates that $-s^{2}$ is a repeated eigenvalue and $\mathbf{a}, \mathbf{b}, \mathbf{c}$ a complete set of eigenvectors of $\mathbf{S}_{1}^{2}$. It should be noted, however, that eigenvectors $\mathbf{a}, \mathbf{b}$ of $\mathbf{S}_{1}^{2}$ corresponding to $-s^{2}$ do not necessarily satisfy Eqs. $(9)_{1,2}$. 
3. Representations of $\mathbf{S}_{\mathbf{2}}, \mathbf{S}_{\mathbf{3}}$, and $\mathbf{Z}$. Equation (12) provides the relations

$$
\mathbf{S}_{1}^{\mathrm{T}} \mathbf{a}^{\prime}=s \mathbf{b}^{\prime}, \quad \mathbf{S}_{1}^{\mathrm{T}} \mathbf{b}^{\prime}=-s \mathbf{a}^{\prime}, \quad \mathbf{S}_{1}^{\mathrm{T}} \mathbf{c}^{\prime}=\mathbf{0},
$$

reciprocal to (9). In combination with (1), rearranged as $\mathbf{S}_{1} \mathbf{S}_{2}=-\mathbf{S}_{2} \mathbf{S}_{1}^{\mathrm{T}}$, (16) and (9) yield

$$
\mathbf{a}^{\prime} \cdot\left(\mathbf{S}_{2} \mathbf{a}^{\prime}\right)=\mathbf{b}^{\prime} \cdot\left(\mathbf{S}_{2} \mathbf{b}^{\prime}\right)=A, \quad \mathbf{a}^{\prime} \cdot\left(\mathbf{S}_{2} \mathbf{b}^{\prime}\right)=0, \quad \mathbf{S}_{2} \mathbf{c}^{\prime}=C \mathbf{c},
$$

where

$$
C=\mathbf{c}^{\prime} \cdot\left(\mathbf{S}_{2} \mathbf{c}^{\prime}\right) .
$$

Constructing the vectors $\mathbf{S}_{2} \mathbf{a}^{\prime}$ and $\mathbf{S}_{2} \mathbf{b}^{\prime}$ from their components relative to the basis $\{\mathbf{a}, \mathbf{b}, \mathbf{c}\}$ we therefore have

$$
\mathbf{S}_{2} \mathbf{a}^{\prime}=A \mathbf{a}, \quad \mathbf{S}_{2} \mathbf{b}^{\prime}=A \mathbf{b}
$$

and, owing to the positive definiteness of $\mathbf{S}_{2}$.

$$
A>0, \quad C>0 .
$$

Equations (18) and (17) ${ }_{4}$ lead to the representation

$$
\begin{aligned}
\mathbf{S}_{2} & =\left(\mathbf{S}_{2} \mathbf{a}^{\prime}\right) \otimes \mathbf{a}+\left(\mathbf{S}_{2} \mathbf{b}^{\prime}\right) \otimes \mathbf{b}+\left(\mathbf{S}_{2} \mathbf{c}^{\prime}\right) \otimes \mathbf{c} \\
& =A(\mathbf{a} \otimes \mathbf{a}+\mathbf{b} \otimes \mathbf{b})+C \mathbf{c} \otimes \mathbf{c}
\end{aligned}
$$

of $\mathbf{S}_{2}$, with inverse

$$
\mathbf{S}_{2}^{-1}=A^{-1}\left(\mathbf{a}^{\prime} \otimes \mathbf{a}^{\prime}+\mathbf{b}^{\prime} \otimes \mathbf{b}^{\prime}\right)+C^{-1} \mathbf{c}^{\prime} \otimes \mathbf{c}^{\prime} .
$$

Introducing the tensor

$$
\mathbf{G}=\mathbf{a} \otimes \mathbf{a}+\mathbf{b} \otimes \mathbf{b}+\mathbf{c} \otimes \mathbf{c},
$$

with determinant

$$
\operatorname{det} \mathbf{G}=g^{2},
$$

we can rewrite Eq. (20) as

$$
\mathbf{S}_{2}=\overline{\mathbf{S}}_{2} \mathbf{G}
$$

where

$$
\overline{\mathbf{S}}_{2}=A\left(\mathbf{a} \otimes \mathbf{a}^{\prime}+\mathbf{b} \otimes \mathbf{b}^{\prime}\right)+C \mathbf{c} \otimes \mathbf{c}^{\prime} .
$$

The right-hand side of (24) is in spectral form, $A$ (twice) and $C$ being eigenvalues and a, $\mathbf{b}, \mathbf{c}$ associated eigenvectors of $\overline{\mathbf{S}}_{2}$. This means that $A$ and $C$ are uniquely determined by $\overline{\mathbf{S}}_{2}$ and it can easily be checked from (10) and (13) that $\mathbf{a} \otimes \mathbf{a}^{\prime}+\mathbf{b} \otimes \mathbf{b}^{\prime}$ and $\mathbf{c} \otimes \mathbf{c}^{\prime}$, in common with $\mathbf{a} \otimes \mathbf{b}^{\prime}-\mathbf{b} \otimes \mathbf{a}^{\prime}$, are independent of $\alpha, \beta, \gamma$. These tensors (but not $\mathbf{G}$ and $\overline{\mathbf{S}}_{2}$ ) are unique in the sense that the triad $\mathbf{a}, \mathbf{b}, \mathbf{c}$ can be chosen arbitrarily from the class defined by (10).

The quantities uniquely determined by $\mathbf{S}_{2}$ are

$$
A(\mathbf{a} \cdot \mathbf{a}+\mathbf{b} \cdot \mathbf{b}), \quad C \mathbf{c} \cdot \mathbf{c}, \quad(\mathbf{a} \cdot \mathbf{a}+\mathbf{b} \cdot \mathbf{b})^{-1}(\mathbf{a} \otimes \mathbf{a}+\mathbf{b} \otimes \mathbf{b}), \quad(\mathbf{c} \cdot \mathbf{c})^{-1} \mathbf{c} \otimes \mathbf{c} .
$$

Clearly this list can be replaced by $A, C, \mathbf{a} \otimes \mathbf{a}+\mathbf{b} \otimes \mathbf{b}, \mathbf{c} \otimes \mathbf{c}$ if $\mathbf{a}, \mathbf{b}, \mathbf{c}$ are normalized so that

$$
\mathbf{a} \cdot \mathbf{a}+\mathbf{b} \cdot \mathbf{b}=1, \quad \mathbf{c} \cdot \mathbf{c}=1
$$


The conditions (25) imply, via (10), that

$$
\alpha=\cos \delta, \quad \beta=\sin \delta, \quad \gamma= \pm 1,
$$

where $\delta$ is arbitrary.

Substitution from (21), (14), and (12) into Eqs. (2) and (3) supplies the representations

$$
\begin{aligned}
\mathbf{S}_{3} & =-A^{-1} F\left(\mathbf{a}^{\prime} \otimes \mathbf{a}^{\prime}+\mathbf{b}^{\prime} \otimes \mathbf{b}^{\prime}\right)-C^{-1} \mathbf{c}^{\prime} \otimes \mathbf{c}^{\prime}, \\
\mathbf{Z} & =A^{-1}\left\{\mathbf{a}^{\prime} \otimes \mathbf{a}^{\prime}+\mathbf{b}^{\prime} \otimes \mathbf{b}^{\prime}+i s\left(\mathbf{a}^{\prime} \otimes \mathbf{b}^{\prime}-\mathbf{b}^{\prime} \otimes \mathbf{a}^{\prime}\right)\right\}+C^{-1} \mathbf{c}^{\prime} \otimes \mathbf{c}^{\prime},
\end{aligned}
$$

of the remaining tensors. It can be verified from Eqs. (13) and (26) that when the normalization (25) applies, the tensors $\mathbf{a}^{\prime} \otimes \mathbf{a}^{\prime}+\mathbf{b}^{\prime} \otimes \mathbf{b}^{\prime}, \mathbf{c}^{\prime} \otimes \mathbf{c}^{\prime}$, and $\mathbf{a}^{\prime} \otimes \mathbf{b}^{\prime}-\mathbf{b}^{\prime} \otimes \mathbf{a}^{\prime}$ are uniquely determined. The symmetric and Hermitian properties of $\mathbf{S}_{3}$ and $\mathbf{Z}$ are evident from (27) and (28) and, by virtue of (19) and (21),

$$
\operatorname{tr} \mathbf{Z}=\operatorname{tr} \mathbf{S}_{2}^{-1}=A^{-1}\left(\mathbf{a}^{\prime} \cdot \mathbf{a}^{\prime}+\mathbf{b}^{\prime} \cdot \mathbf{b}^{\prime}\right)+C^{-1} \mathbf{c}^{\prime} \cdot \mathbf{c}^{\prime}>0
$$

(cf. $[4$, Sec. 3(a)]).

In parallel with the decomposition (23),

$$
\mathbf{S}_{3}=\mathbf{G}^{-1} \overline{\mathbf{S}}_{3},
$$

where

$$
\overline{\mathbf{S}}_{3}=-A^{-1} F\left(\mathbf{a} \otimes \mathbf{a}^{\prime}+\mathbf{b} \otimes \mathbf{b}^{\prime}\right)-C^{-1} \mathbf{c} \otimes \mathbf{c}^{\prime} .
$$

We recognize here a third spectral form, and the coaxiality of $\mathbf{S}_{1}^{2}, \overline{\mathbf{S}}_{2}$, and $\overline{\mathbf{S}}_{3}$ is displayed by the connections

$$
\overline{\mathbf{S}}_{2}=C \mathbf{I}-(1-F)^{-1}(A-C) \mathbf{S}_{1}^{2}, \quad \overline{\mathbf{S}}_{3}=-C^{-1} \mathbf{I}+(1-F)^{-1}\left(A^{-1} F-C^{-1}\right) \mathbf{S}_{1}^{2},
$$

resulting from Eqs. (14), (24), and (29).

The products

$$
\begin{aligned}
& \mathbf{S}_{1} \mathbf{S}_{2}=s A(\mathbf{a} \otimes \mathbf{b}-\mathbf{b} \otimes \mathbf{a}), \\
& \mathbf{S}_{3} \mathbf{S}_{1}=-s A^{-1} F\left(\mathbf{a}^{\prime} \otimes \mathbf{b}^{\prime}-\mathbf{b}^{\prime} \otimes \mathbf{a}^{\prime}\right)
\end{aligned}
$$

of the representations (12), (20), and (27) show that $\mathbf{S}_{3} \mathbf{S}_{1}$, like $\mathbf{S}_{1} \mathbf{S}_{2}$, is skew-symmetric. The axial vectors of $\mathbf{S}_{1} \mathbf{S}_{2}$ and $\mathbf{S}_{3} \mathbf{S}_{1}$ are $-s g A \mathbf{c}^{\prime}$ and $s g^{-1} A^{-1} F \mathbf{c}$, respectively.

Bounds can be set for $F$, and hence for $s$, with the aid of (15) $)_{1}$ and the relation

$$
F=\left\{\mathbf{a}^{\prime} \cdot\left(\mathbf{S}_{2} \mathbf{a}^{\prime}\right)\right\}\left\{\mathbf{a} \cdot\left(-\mathbf{S}_{3} \mathbf{a}\right)\right\},
$$

arising from (17) $)_{1,2}$ and (27). If $\boldsymbol{\sigma}$ and $\tau$ are the smallest eigenvalues of $\mathbf{S}_{2}$ and $-\mathbf{S}_{3}$, respectively,

$$
\boldsymbol{\sigma} \tau|\mathbf{a}|^{2}\left|\mathbf{a}^{\prime}\right|^{2} \leqslant F<1
$$

and since $|\mathbf{a}|^{2}\left|\mathbf{a}^{\prime}\right|^{2} \geqslant\left(\mathbf{a} \cdot \mathbf{a}^{\prime}\right)^{2}=1,(30)$ simplifies to

$$
\sigma \tau \leqslant F<1
$$

when $\tau \geqslant 0$.

4. Discussion and additional relations. The representations (12), (20), (27), and (28) are of striking simplicity, only three scalars, $s, A, C$, being involved in addition to the basis $\{\mathbf{a}, \mathbf{b}, \mathbf{c}\}$ and its reciprocal. Since $s$ is dimensionless while $A$ and $C$ have the physical dimensions of (stress) $)^{-1}$, the number of independent scalars cannot be less than two. 
Plainly, then, the structure of the Barnett-Lothe and impedance tensors is only slightly influenced by the symmetry of the elastic material forming $\mathscr{B}$.

Besides revealing the essential form and interrelationship of $\mathbf{S}_{1}, \mathbf{S}_{2}, \mathbf{S}_{3}$, and $\mathbf{Z}$, the results of Secs. 2 and 3 serve to expedite the evaluation of the tensors. Suppose that $\mathbf{S}_{1}$ and $\operatorname{tr} \mathbf{S}_{2}$ have been found, either algebraically or from their integral representations [3, Sec. IV, D]. Then $s$ and $\mathbf{a}, \mathbf{b}, \mathbf{c}$ can be derived from (8) and $\mathbf{a}^{\prime}, \mathbf{b}^{\prime}, \mathbf{c}^{\prime}$ follow from (11). If the scalar $\mathbf{c}^{\prime} \cdot\left(\mathbf{S}_{2} \mathbf{c}^{\prime}\right)$ is now determined, by either of the aforementioned methods, $(17)_{5}$ and the relation

$$
\operatorname{tr} \mathbf{S}_{2}=A+C,
$$

afforded by (20) and (25), fix the remaining scalars $A$ and $C$. The use of Eqs. (20), (27), and (28) completes the calculation.

The application of this procedure to formulas for an isotropic elastic body given in [3, Sec. VI, E] yields the expressions

$$
\begin{aligned}
& s=\left(\gamma^{2} \xi \eta\right)^{-1 / 2}(2-\gamma-2 \xi \eta), \quad F=-\left(\gamma^{2} \xi \eta\right)^{-1}(1-\xi \eta)\left\{(2-\gamma)^{2}-4 \xi \eta\right\}, \\
& A=(\mu \gamma \xi \eta)^{-1}(\xi+\eta)(1-\xi \eta), \quad C=(\mu \eta)^{-1}, \\
& \mathbf{a}=-\{\eta /(\xi+\eta)\}^{1 / 2} \mathbf{e}_{1}, \quad \mathbf{b}=\{\xi /(\xi+\eta)\}^{1 / 2} \mathbf{e}_{2}, \quad \mathbf{c}=\mathbf{e}_{3} .
\end{aligned}
$$

Here

$$
\gamma=\left(\rho v^{2} / \mu\right)^{1 / 2}, \quad \xi=(1-\Lambda \gamma)^{1 / 2}, \quad \eta=(1-\gamma)^{1 / 2}, \quad \Lambda=\mu /(\lambda+2 \mu),
$$

$\lambda$ and $\mu$ being the Lamé constants of the material and $\mathbf{e}_{i}$ the unit vector in the $x_{i}$-direction. There are thus only two independent scalars in this case and the vectors $\mathbf{a}, \mathbf{b}$, c are mutually orthogonal.

Setting $\nu= \pm i$ in equation (5) $)_{1}$ and using (15) $)_{1.2}$ we obtain

$$
\operatorname{det}\left(\mathbf{I} \pm i \mathbf{S}_{1}\right)=F .
$$

Then, from Eqs. (2) and (3),

$$
\operatorname{det} \mathbf{S}_{3}=-\left(\operatorname{det} \mathbf{S}_{2}\right)^{-1} F^{2}, \quad \operatorname{det} \mathbf{Z}=\left(\operatorname{det} \mathbf{S}_{2}\right)^{-1} F,
$$

and, from (22) to (24),

$$
\operatorname{det} \mathbf{S}_{2}=g^{2} A^{2} C \text {. }
$$

Equations (35) to (37) tell us that $\mathbf{I} \pm i \mathbf{S}_{1}, \mathbf{S}_{3}$, and $\mathbf{Z}$ are nonsingular for all values of $v \in I$ for which $F \neq 0$. The inverses can be found with the help of the equality

$$
\mathbf{S}_{1}^{3}+(1-F) \mathbf{S}_{1}=\mathbf{0},
$$

derived from $(5)_{2}$ and (15) $)_{1,2}$ by applying the Cayley-Hamilton theorem. They are

$$
\begin{gathered}
\left(\mathbf{I} \pm i \mathbf{S}_{1}\right)^{-1}=\mathbf{I} \mp i F^{-1} \mathbf{S}_{1}-F^{-1} \mathbf{S}_{1}^{2}, \\
\mathbf{S}_{3}^{-1}=-\left(\mathbf{I}-F^{-1} \mathbf{S}_{1}^{2}\right) \mathbf{S}_{2}, \quad \mathbf{Z}^{-1}=-\mathbf{S}_{3}^{-1}-i F^{-1} \mathbf{S}_{1} \mathbf{S}_{2},
\end{gathered}
$$


and we also have

$$
\left(\mathbf{I}+\mathbf{S}_{1}^{2}\right)^{-1}=\mathbf{I}-F^{-1} \mathbf{S}_{1}^{2} .
$$

In view of (4) ${ }_{2}$, Eqs. (2), (3), and (38) imply that each of the symmetric tensors $\mathbf{S}_{2}^{-1}+\mathbf{S}_{3}$, $\mathbf{S}_{2}+\mathbf{S}_{3}^{-1}$ and each of the Hermitian tensors $\mathbf{Z}-\mathbf{S}_{2}^{-1}, \mathbf{Z}^{-1}-\mathbf{S}_{2}, \mathbf{Z}+\mathbf{S}_{3}, \mathbf{Z}^{-1}+\mathbf{S}_{3}^{-1}$ is singular. Moreover, $\mathbf{Z}-\mathbf{S}_{2}^{-1}$ and $\mathbf{Z}^{-1}+\mathbf{S}_{3}^{-1}$ are imaginary and skew-symmetric, and therefore traceless.

Let $\mathbf{u}$ and $\mathbf{v}$ be arbitrary nonzero vectors, $\mathbf{u}$ real and $\mathbf{v}$ complex, with real and imaginary parts $\mathbf{v}^{+}, \mathbf{v}^{-}$. In view of the inequalities (19) the identities

$$
\begin{aligned}
\mathbf{u} \cdot( & \left.-\mathbf{S}_{3} \mathbf{u}\right)=A^{-1} F\left\{\left(\mathbf{u} \cdot \mathbf{a}^{\prime}\right)^{2}+\left(\mathbf{u} \cdot \mathbf{b}^{\prime}\right)^{2}\right\}+C^{-1}\left(\mathbf{u} \cdot \mathbf{c}^{\prime}\right)^{2}, \\
\overline{\mathbf{v}} \cdot(\mathbf{Z v})= & A^{-1}\left[\left(\mathbf{v}^{+} \cdot \mathbf{a}^{\prime}-s \mathbf{v}^{-} \cdot \mathbf{b}^{\prime}\right)^{2}+\left(\mathbf{v}^{+} \cdot \mathbf{b}^{\prime}+s \mathbf{v}^{-} \cdot \mathbf{a}^{\prime}\right)^{2}\right. \\
& \left.+F\left\{\left(\mathbf{v}^{-} \cdot \mathbf{a}^{\prime}\right)^{2}+\left(\mathbf{v}^{-} \cdot \mathbf{b}^{\prime}\right)^{2}\right\}\right]+C^{-1}\left\{\left(\mathbf{v}^{+} \cdot \mathbf{c}^{\prime}\right)^{2}+\left(\mathbf{v}^{-} \cdot \mathbf{c}^{\prime}\right)^{2}\right\},
\end{aligned}
$$

formed from Eqs. (27), (28), and (15), prove that $F>0, F=0$, and $F<0$ are, in turn, necessary and sufficient conditions for $-\mathbf{S}_{3}$ and $\mathbf{Z}$ to be positive definite, positive semidefinite, and indefinite.

If strong ellipticity of the linear elasticity tensor is replaced by the more stringent requirement that the strain energy of the material be positive definite, $-\mathbf{S}_{3}$ is positive definite at $v=0[3, \mathrm{Sec} . \mathrm{V}, \mathrm{C}, 2]$. Furthermore, subject to the strong ellipticity condition, the derivative of $\mathbf{S}_{3}$ with respect to $v$ is positive definite in $I$ [7, Sec. 3(d)], implying that the eigenvalues of $\mathbf{S}_{3}$ increase monotonically with $v$ ([8] and [3, Sec. VIII,A,1]). If $F$ has a zero, $v_{s}$ say, in $I$, inspection of Eq. (27) shows that $\mathbf{S}_{3}$ has eigenvalues 0 (twice) and $-C^{-1} \mathbf{c}^{\prime} \cdot \mathbf{c}^{\prime}$ at $v=v_{s}$. This has two consequences. First, $v_{s}$ is unique since otherwise at least one eigenvalue would vanish twice in $I$, contrary to the monotonicity property. Second, $-\mathbf{S}_{3}$ has two negative eigenvalues for $v_{s}<v<\hat{v}$, thus limiting the validity of the bounds (31) to the interval $\left[0, v_{s} \text { ). When it exists } v_{s} \text { is seen from Eq. (36) }\right)_{2}$ to be a zero of $\operatorname{det} \mathbf{Z}$ as well as $F$ and $\operatorname{det} \mathbf{S}_{3}$. In fact $v_{s}$ is the speed with which a subsonic surface wave propagates in the $x_{1}$-direction in a semi-infinite body with traction-free boundary given by $x_{2}=0$, and $\mathbf{a}+i \mathbf{b}$, evaluated at $v=v_{s}$, is the polarization vector of the wave [3, Sec. VII,A,B,D].

The foregoing arguments establish $F=0$ as the fundamental form of the secular equation for an elastic surface wave (cf. [9, Sec. 3.1]) and prove that $F$ lies between 0 and 1 in $\left[0, v_{s}\right)$ and is negative in $\left(v_{s}, \hat{v}\right)$. When the material is isotropic, $\hat{v}=(\mu / \rho)^{1 / 2}$ and Eq. $(32)_{2}$, with $(34)_{3,4}$, confirms that $v_{s}$ is the unique positive real root of Rayleigh's equation

$$
(2-\gamma)^{2}=4(1-\gamma)^{1 / 2}(1-\Lambda \gamma)^{1 / 2} \text {. }
$$

We have concentrated above on the representations (12), (20), (27), and (28) and such of their implications as seem significant to us. The results by no means exhaust the relations that could be written down and, to conclude this section, we quote as examples

$$
\mathbf{S}_{1} \mathbf{S}_{2} \mathbf{S}_{3}=\mathbf{S}_{2} \mathbf{S}_{3} \mathbf{S}_{1}=-F \mathbf{S}_{1}
$$

and

$$
\mathbf{S}_{3}=-\mathbf{Z S}_{2} \mathbf{Z}^{\mathrm{T}}=-\mathbf{Z}^{\mathrm{T}} \mathbf{S}_{2} \mathbf{Z}
$$


Recalling the properties of $\mathbf{S}_{1} \mathbf{S}_{2}$ and $\mathbf{S}_{3} \mathbf{S}_{1}$ stated in Sec. 3, we deduce from (39) that the axial vectors of the skew-symmetric tensors $\mathbf{S}_{2}^{-1} \mathbf{S}_{1}$ and (when $\left.v \neq v_{s}\right) \mathbf{S}_{1} \mathbf{S}_{3}^{-1}$ are $-s g^{-1} A^{-1} \mathbf{c}$ and $s g A F^{-1} \mathbf{c}^{\prime}$, respectively.

5. Invariance under rotations about the $x_{3}$-axis. Intrinsic to the calculation of $\mathbf{S}_{1}, \mathbf{S}_{2}, \mathbf{S}_{3}$ is the orthonormal basis $e=\left\{\mathbf{e}_{1}, \mathbf{e}_{2}, \mathbf{e}_{3}\right\}$ introduced in connection with Eqs. (33). The vector $\mathbf{e}_{1}$ represents the direction of steady motion (when $v>0$ ) and $\mathbf{e}_{3}$ is normal to the planes which are equivalent in the motion. The basis as a whole provides the three-dimensional reference frame needed to define the orientation of the axes (or other elements) of material symmetry.

Let $\hat{e}=\left\{\hat{\mathbf{e}}_{1}, \hat{\mathbf{e}}_{2}, \hat{\mathbf{e}}_{3}\right\}$ be the orthonormal basis obtained by rotating $e$ through an angle $\theta(0 \leqslant \theta<2 \pi)$ about $\mathbf{e}_{3}$. We denote by $\Omega=\hat{\mathbf{e}}_{p} \otimes \mathbf{e}_{p}$ (with summation on $p$ ) the proper orthogonal tensor which carries $e$ into $\hat{e}$ and systematically place caps above quantities evaluated with $\hat{e}$ as reference frame.

Transformation rules satisfied by the eigenvectors of the eigenvalue problem of plane anisotropic elastostatics under rotations of the reference frame which preserve the equivalent planes have been derived by Ting [10, Sec. 3]. These formulae, in conjunction with the algebraic representations of $\left.\mathbf{S}_{1}, \mathbf{S}_{2}, \mathbf{S}_{3}[3 \text {, Eqs. (4.32) })_{1},(4.33)_{1},(4.34)_{1}\right]$, show that, when $v=0$,

$$
\hat{\mathbf{S}}_{k}=\Omega_{\mathbf{S}_{k}} \Omega^{\mathrm{T}}, \quad k=1,2,3 .
$$

Equations (40) may also be obtained directly from the integral representations of $\mathbf{S}_{1}, \mathbf{S}_{2}$, $\mathbf{S}_{3}\left[3\right.$, Eqs. $\left.(4.32)_{2},(4.33)_{2},(4.34)_{2}\right]$ by means of the easily established properties

$$
\hat{\mathbf{Q}}(\Omega \mathbf{n})=\Omega \mathbf{Q}(\mathbf{n}) \Omega^{\mathrm{T}}, \quad \hat{\mathbf{R}}(\Omega \mathbf{m}, \Omega \mathbf{n})=\Omega \mathbf{R}(\mathbf{m}, \mathbf{n}) \Omega^{\mathrm{T}}
$$

of the acoustical and associated acoustical tensors.

We infer from (40) that, when $v=0$, the eigenvalues of the Barnett-Lothe tensors are invariant under the transformation $e \rightarrow \hat{e}$. In particular, $\hat{s}=s$ and, from $(15)_{1}, \hat{F}=F$. It then follows from Eq. (8) that

$$
\hat{\mathbf{a}}=\Omega(\phi \mathbf{a}-\chi \mathbf{b}), \quad \hat{\mathbf{b}}=\Omega(\chi \mathbf{a}+\phi \mathbf{b}), \quad \hat{\mathbf{c}}=\psi \Omega \mathbf{c},
$$

where $\phi, \chi, \psi$ are arbitrary real numbers such that $\phi^{2}+\chi^{2} \neq 0, \psi \neq 0$. Since a proper orthogonal tensor is self-adjugate [6, p. 20] Eqs. (11) provide the corresponding formulae

$$
\begin{aligned}
& \hat{\mathbf{a}}^{\prime}=\left(\phi^{2}+\chi^{2}\right)^{-1} \Omega\left(\phi \mathbf{a}^{\prime}-\chi \mathbf{b}^{\prime}\right), \\
& \hat{\mathbf{b}}^{\prime}=\left(\phi^{2}+\chi^{2}\right)^{-1} \Omega\left(\chi \mathbf{a}^{\prime}+\phi \mathbf{b}^{\prime}\right), \\
& \hat{\mathbf{c}}^{\prime}=\psi^{-1} \Omega \mathbf{c}^{\prime}
\end{aligned}
$$

for the reciprocal vectors. If the normalization (25) is imposed in both $e$ and $\hat{e}$ the restrictions

$$
\phi^{2}+\chi^{2}=1, \quad \psi^{2}=1
$$

apply to (41) and (42). (The stronger conditions $\phi=1, \chi=0, \psi=1$ may be obtained by requiring the triads $\{\mathbf{a}, \mathbf{b}, \mathbf{c}\}$ and $\{\hat{\mathbf{a}}, \hat{\mathbf{b}}, \hat{\mathbf{c}}\}$ to be congruent in the sense that scalar products and the scalar triple product are unchanged by the rotation $e \rightarrow \hat{e}$.) Lastly, the application of (40) and (42), with (43), to the definitions of $A$ and $C$ in (17) gives $\hat{A}=A, \hat{C}=C$. 
When $v=0$ and the normalization (25) is applied uniformly the scalars $s, F, A, C$ appearing in the representations (12), (20), (27), and (28) are therefore invariants under an arbitrary rotation of the reference frame about the $x_{3}$-axis. The vectors $\mathbf{a}, \mathbf{b}, \mathbf{c}$ obey the transformation rules

$$
\hat{\mathbf{a}}=\Omega(\cos \varepsilon \mathbf{a}-\sin \varepsilon \mathbf{b}), \hat{\mathbf{b}}=\Omega(\sin \varepsilon \mathbf{a}+\cos \varepsilon \mathbf{b}), \quad \hat{\mathbf{c}}= \pm \Omega \mathbf{c},
$$

where $\varepsilon$ is arbitrary, and the reciprocal vectors $\mathbf{a}^{\prime}, \mathbf{b}^{\prime}, \mathbf{c}^{\prime}$ transform in the same way. When $\{\mathbf{a}, \mathbf{b}, \mathbf{c}\}$ is required to remain congruent, however, $\varepsilon=0$ and the ambiguity of sign in $(44)_{3}$ disappears.

When $v>0, \mathbf{e}_{1}$ assumes the role of a preferred direction since, in relation to $\hat{e}$, $\cos \theta \hat{\mathbf{e}}_{1}-\sin \theta \hat{\mathbf{e}}_{2}$, and not $\hat{\mathbf{e}}_{1}$, is the unit vector in the direction of steady motion. The simplicity of behavior under rotations about the $x_{3}$-axis encountered when $v=0$ is consequently lost.

Acknowledgments. The second author (TCTT) wishes to acknowledge support from the U.S. Army Research Office through grant DAAG 29-84-K-0159 to the University of Illinois at Chicago.

Addendum. Since the acceptance of this paper we have seen a note by Kirchner and Lothe [11] showing, by an approach different to that followed here, how $\mathbf{S}_{1}$ and $\mathbf{S}_{3}$ (or $\mathbf{S}_{1}$ and $\mathbf{S}_{2}$ ) can be calculated when $\mathbf{S}_{2}$ (or $\mathbf{S}_{3}$ ) is known together with the acoustical and associated acoustical tensors $\mathbf{Q}$ and $\mathbf{R}$ appearing in the integral representations of $\mathbf{S}_{1}, \mathbf{S}_{2}$, and $\mathbf{S}_{3}$ mentioned in Section 4.

\section{REFERENCES}

[1] D. M. Barnett and J. Lothe, Synthesis of the sextic and integral formalism for dislocation:, Greens functions and surface waves in anisotropic elastic solids, Phys. Norv. 7, 13-19 (1973)

[2] K. A. Ingebrigtsen and A. Tonning, Elastic surface waves in crystals, Phys. Rev. 184, 942-951 (1969)

[3] P. Chadwick and G. D. Smith, Foundations of the theory of surface waves in anisotropic elastic materials, Advances in Applied Mechanics (Ed. C.-S. Yih), Vol. 17, Academic Press, New York, 303-376, 1977

[4] D. M. Barnett and J. Lothe, Free surface (Rayleigh) waves in anisotropic elastic half-spaces: the surface impedance method, Proc. Roy. Soc. Lond. A402, 135-152 (1985)

[5] T. C. T. Ting, Explicit solution and invariance of the singularities at an interface crack in anisotropic composites, Internat. J. Solids and Structures 22, 965-983 (1986)

[6] P. Chadwick, Continuum mechanics. Concise theory and problems, Allen \& Unwin, London, 1976

[7] P. Chadwick and D. A. Jarvis, Surface waves in a prestressed elastic body, Proc. Roy. Soc. Lond. A366, 517-536 (1979)

[8] D. M. Barnett, J. Lothe, K. Nishioka, and R. J. Asaro, Elastic surface waves in anisotropic crystals: A simplified method for calculating Rayleigh velocities using dislocation theory, J. Phys. F. 3, 1083-1096 (1973)

[9] P. Chadwick and G. D. Smith, Surface waves in cubic elastic materials. Mechanics of Solids, The Rodney Hill 60th Anniversary Volume (eds, H. G. Hopkins and M. J. Sewell), Pergamon, Oxford, 47-100, 1982

[10] T. C. T. Ting, Effects of change of reference coordinates on the stress analyses of anisotropic elastic materials. Internat. J. Solids and Structures 18, 139-152 (1982)

[11] H. O. K. Kirchner and J. Lothe, On the redundancy of the $\bar{N}$ matrix of anisotopic elasticity, Phil. Mag. A53, L7-L10 (1986) 